tions of stereographic and gnomonic projections. However, in relation to these projections, he uses only the polar angle, which he does not name and which he calls $\theta$ instead of $\varrho$, and he makes no reference to an azimuthal angle. The fuller description is of the stereographic projection rather than the gnomonic, and he uses the former throughout the rest of the book. The explanation of face intercepts and Miller indices is fairly satisfactory although the interrelationship between unit-cell dimensions, axial ratios, and Miller indices might be difficult for the beginner to understand from this treatment. Chapter 3 concludes with an explanation of how the stereographic projection of a crystal is drawn up with the aid of a Wulff net utilizing sets of interfacial angles taken in zones.

Part 2, Systematic Crystallography, is generally more satisfactory than part 1 . It consists essentially of the traditional morphological descriptions of crystals taken by crystal class within the systems taken in the sequence tetragonal, or- thorhombic, monoclinic, triclinic, cubic, and hexagonal and trigonal. In an introductory text such as this, the full Hermann-Mauguin class symbols $(4 / \mathrm{m}$ $2 / m 2 / m$ ) are to be preferred to the shortened symbols $(4 / \mathrm{mmm})$. Well executed drawings show for each class the symmetry elements, a stereogram of the symmetry, the individual crystal forms, and the forms and habits of some representative crystals. The representative crystal drawings would be enhanced by the inclusion of the reference axes (which are shown on the drawings of the individual forms). In the text, some explanation of the origin or meaning of unfamiliar crystal form names would be helpful to the beginning crystallographer. Each chapter dealing with one crystal system concludes with a detailed account of the procedure for drawing a stereographic projection of a crystal in that system, deducing its symmetry, deriving an axial ratio, and indexing the faces. These sections along with the last two chapters of the book, 12, Stereographic Projections - Some Ad- ditional Constructions, and 13, Crystal Drawing, are perhaps the most valuable in the whole book. The main part of the text concludes with chapter 11, Crystal Growth and Composite Crystals, which includes a treatment of twinning appropriate to the scope of the book although the term 'twin law' is used only once.

An Outline of Crystal Morphology hardly does justice to the structural principles underlying crystal morphology, but it does give a reasonably satisfactory account of crystal morphology itself including the stereographic representation of crystals, and the drawing of crystal forms. It should be found useful by professional crystallographers with an interest in morphology, and by amateur crystallographers and mineralogists.

R. B. Ferguson

Department of Earth Sciences

The University of Manitoba

Winnipeg 19

Canada

\section{Meeting Reports}

\section{Iber-American Group on \\ Crystallography. Second \\ Meeting, 8-17 June 1970.}

\section{Background}

That the development of the different branches of science and technology should be closely entwined with the necessities and possibilities of each geopolitical area is at present an accepted fact. It is also accepted that this development demands that the teaching and research should be oriented accordingly to original strategies. The planning of these strategies, however, is not as obvious as it may appear and it becomes particularly difficult in those countries where there are not a great many scientists. There, the scientific research is carried on by small groups of scientists who, for the most part, have been trained in other countries with cultural, political and economic structures different from those of their own. While these groups maintain close relationships with foreign groups working in totally different environments, they tend to have little contact with those researchers working in surroundings similar to theirs. Evidently, this fact is a hindrance to the development of the adequate patterns and strategies to be followed in each particular environment, which otherwise ought to develop from common and cooperative efforts of groups working in related areas of research under like difficulties.

In this sense, regional meetings appear as very important, since they bring into contact scientists from countries with similar conditions. The Iber-American countries provide an interesting example: the existing bonds among scientists, quite strong among these countries, are based more on the similarity of economic-political situations and cultural backgrounds than on the mere affinity of languages.

On the basis of these ideas, a small number of researchers decided to form an Iber-American Association in crystallography. The Association was constituted in the city of Cordoba, Argentina, during the 36th Congress of the Argentine Physical Society in 1960 , which was attended by crystallographers of several Iber-American countries.

\section{Second meeting}

In August 1969, during the Eighth International Congress of Crystallography held in Stony Brook, the founders of the Iber-American branch met again and programmed a second meeting which took place in Buenos Aires during June 8-17, 1970. The chairman of the organizing committee was Professor E.
Galloni. The programming and organizing of this meeting took into special account the particular conditions of the participating countries. In this regard, one of the most important points to be considered was that in the Iber-American countries there are too few people working in each research area to permit the organization of a Congress devoted mainly to original papers; ordinarily, the few people working in a given area already will have had occasion to become aware of each other's new results. An analysis of the short and medium range possibilities of their respective areas was, instead, presented by several lberAmerican specialists.

The meeting began with a general survey by Professor Julio Garrido from Spain. R. Bru (University of Madrid) and $M$. Ipohorski (Argentine Atomic Energy Commission) presented the latest achievements in and possibilities of electron microscopy. OI Wittke (University of Chile) did the same with lattice dynamics studies. A. Bonfiglioli (Argentine Atomic Energy Commission) analysed diffraction by imperfect crystals and $\mathrm{E}$. Laredo (Venezuelan Institute of Scientific Research) presented a review of small angle $X$-ray scattering. $L$. Levy and P. Diodati (University of Buenos Aires) reviewed crystal growth tech- 
niques and electron diffraction by gases. E. Manghi (Scientific Research Laboratory of the Armed Forces, Argentina) reviewed the possibilities of $X$-ray topography.

The meeting was complemented by two short courses (4 lectures each). The one on progress in the determination of crystalline structures was conclucted by L. Becka (Johns Hopkins University) and S. Baggio (Buenos Aires University). The one on anomalous scattering was conducted by S. Caticha-Ellis (Atomic Energy Research Center, São Paulo, Brazil).

Finally, two panels met. One was on the teaching of crystallography. The other, on instrumentation, placed particular emphasis on the existence and distribution of various equipment in the Iber-American countries.

In parallel with the activities cited, ad hoc sessions were held to discuss original papers and visits were made to different laboratories.

In addition to the obvious benefits resulting from the interchange of information among scientists who will perform their activities in countries of similar environment, a strong relationship was initiated among different working groups within the Iber-American area. It is hoped that this relationship will be significantly strengthened, through cooperation in programs of research and teaching, by the time of the next meeting now being planned for 1972 at Valdivia, Chile.

Alberto Bonfigliol

Gerencia de Tecnología

Comisión Nacional de Energía Atómica

Avda. del Libertador 8250

Buenos Aires

Argentina

\section{Highlights of the Ottawa Meeting of the American Crystallographic Associa- tion. Carleton University, 16 - 22 August 1970.}

$X$-ray interferometry has largely developed out of the pioneering work of Bonse (Munster) and Hart (Bristol). This work resulted in their receiving the first B. E. Warren Award of the American Crystallographic Association at Ottawa. Bonse and Hart surveyed the present status of $X$-ray interferometry in their two invited papers. Use of $X$-rays rather than visible light for interferometry, with the resulting increase in accuracy commensurate with the decrease in wavelength, has always been possible in principle. Practical X-ray interferometry has only recently become possible with the availability of large, nearly perfect, crystals such as those of silicon. Coherent wavefields, modulated with the periodicity of the crystal lattice, can thereby be produced. The severe problem of aligning the interferometer elements within a fraction of an angstrom precision was solved by using one large single crystal into which thin slices are machined and etched. An integral interferometer unit, preserving the coherence of the single crystal lattice, may thus be made.

Bonse and Hart showed that variations in lattice constants, due to variations of impurities or point defect concentrations, can be detected interferometrically with a sensitivity of better than one part in $10^{8}$. Dispersion corrections can be measured directly with an accuracy of 0.1 per cent, and lattice spacings can be determined with an absolute accuracy of a few parts in $10^{7}$, by direct comparison with optical wavelengths.

$\mathrm{X}$-ray topography has been used by Patel (Murray Hill) to study magnetic domains in orthoferrites, made visible by magnetostrictive distortion of the crystals. Cockayne and Ray (Oxford) described the use of weakly diffracting beams to produce transmission electron microscopy images of extended dislocations. Closely spaced line pairs corresponding to partial dislocations were observed in a variety of crystals including, rather surprisingly, several covalent crystals. Cowley (Tempe) discussed a new method for deriving information about the spatial distribution of potential for excited states in crystals using inelastic electron diffraction.

Several papers were concerned with the most efficient use of small computers dedicated to the control of automatic diffractometers. So far, such use concentrates on the 'production' of intensity data and neglects the subtler but more challenging aspects of 'quality control' of the data being produced.

At the last previous A.C.A. meeting (New Orleans), Hauptman (Buffalo) had presented a new structure invariant formula with exciting possibilities for phase determination. In Ottawa, Hauptman demonstrated the power of this formula, and Duax (Buffalo) described its use in solving the structure of an estradiol-urea complex. The application of normal probability plot analysis to data measured on two crystals of LuBO was discussed by Bernstein et al. (Murray
Hill): the standard deviations in atomic coordinates derived by least-squares from the inverse matrix of the normal equations were conclusively shown to be too small, in this case by as much as 50 per cent.

Lipscomb (Cambridge) reviewed the current methods and results of protein crystallography: 23 protein structures have now been studied at high resolution, 5 at moderate and at least 9 at low resolution. The method of isomorphous replacement remains the most effective tool in the elucidation of protein structures. The least-squares and difference Fourier-series refinement of rubredoxin at $1.5 \AA$, or almost atomic, resolution by Watenpaugh et al. (Seattle), represents the first such successful refinement on a protein structure. Rigorous 'small molecule' crystallographic procedures were applied in this study, and were reported to lead to an overall $R$ value of 0.179 . The least-squares refinement did not diverge, as is generally found to occur with proteins at initial $R$ values of about $0 \cdot 4$. Similar procedures must doubtless be used in all protein structure studies.

A total of 65 papers were devoted to organic crystal structure determination, including organo-metallic and coordination compounds, and 32 to inorganic crystal structure determination, including minerals. The primary interest in these papers was in understanding the physical or chemical properties of the materials, rather than their crystallography. Five of the organic structures discussed were disordered. Such structures are by no means uncommon, but they do present unusual difficulty in interpretation and refinement. It is likely that many have hitherto gone unreported. Advanced crystallographic procedures are now widely enough used that the number of reports on disordered organic structures may approach the actual frequency of occurrence. The ordered structures of small molecules of biological interest are now often quickly and routinely solved by direct methods applied to relatively high quality diffractometer data. Typical structures thus solved are the antitumor agent $\mathrm{C}_{19} \mathrm{H}_{27} \mathrm{~N}_{3} \mathrm{O}_{2} \mathrm{Cl}$ reported by Berman and coworkers (Philadelphia) and the teratogen thalidomide $\mathrm{C}_{13} \mathrm{H}_{10} \mathrm{~N}_{2} \mathrm{O}_{4}$ by both Lovell (New York) and Allen and Trotter (Vancouver).

The same methods are equally successful in solving structures such as the organometallic complexes of the transition metals. A propeller-shaped molecule has thereby been reported by Birnbaum (Ottawa) for an iron tricarbonyl 
complex with 12-oxa[4,4,3]propella$2,4,7,9$ tetraene. Structural studies on purine-pyrimidine complexes gave Sobell and coworkers (Rochester) important new information on base pairing in DNA and RNA. In eight out of nine complexes of adenine and thymine (uracil), the 'Hoogsteen' or reversed 'Hoogsteen' type of hydrogen bonding was found which is not the Watson and Crick pairing scheme for DNA.

The inorganic structure papers provided considerable information, especially on phase transitions, on order-disorder, and on conformation. The characterization of two more low temperature phases in the $X_{e} F_{6}$ system, based on $\mathrm{XeF}_{5}^{+}$and $\mathrm{F}^{-}$ion association, was reported by Jones \& Burbank (Murray Hill), and Peterson and coworkers (Argonne) found an $\mathrm{XeF}_{8}^{2-}$ ion with a distorted Archimedean antiprismatic configuration. A model for the complex ferroelectric ferroelastic-paraelectric paraelastic phase transformation in $\beta-\mathrm{Gd}_{2}\left(\mathrm{MoO}_{4}\right)_{3}$ was discussed by Keve and co-workers (Murray Hill), and Larson \& Cromer (Los Alamos) presented one of the first, if not the first, successful applications of symbolic addition procedures to data for a cubic crystal. Roth (Schenectady) showed the high conductivity of the silver isomorph of $\beta$-alumina to be due to a disordered structure with $\mathrm{Ag}$ ions essentially randomly distributed in channels in the structure.

Abstracts of the meeting are available from Polycrystal Book Service, P.O. Box 11567, Pittsburgh, Pa. 15238 at $\$ 12.25$ postpaid. This report is based on notes kindly provided by the following session chairmen: F. R. Ahmed, P. Coppens, R. D. Burbank, W. C. Hamilton, J. M. Cowley, A. W. Hanson, A. C. Larson, L. H. Jensen, J. Trotter and C. N. Caughlan, whom it is a pleasure to thank.

S. C. Abrahams

Bell Telephone Laboratories, Inc. Murray Hill

New Jersey

U.S.A.

\section{The Second National Confe- rence of Crystal Growth in Japan. Held at Nagoya, 9-10 November 1970.}

This conference was organized by the Japan Society of Applied Physics under the co-sponsorship of other nine academic societies in Japan. Four invited lectures and 57 contributed papers were read in 8 sessions. About 500 people from universities, industries and research laboratories attended the conference.

The four invited lectures were:

T. Arizumi and T. Nishinaga (Nagoya University) Vapor growth of the group III-V compounds and their alloys.

Shin. Takasu, H. Takei, T. Fukuda and S. Matsumura (Tokyo Shibaura Electric Co.). The growth conditions of opto-electronic crystals.

I. Nakata (University of Tokyo). Some problems of crystal growth in liquid crystals.

K. Kinosita and K. Takeuchi (Gakushuin University). Initial stages of thin film growth.

The materials in the contributed papers are as follows:

Session I. Vapor growth (12 papers)

Growth of $\mathrm{CdS}, \beta-\mathrm{SiC}, \mathrm{Ni}_{3} \mathrm{~B}_{7} \mathrm{O}_{13} \mathrm{l}$ $\mathrm{CdCr}_{2} \mathrm{~S}_{4}, \mathrm{Cu}_{2} \mathrm{M}_{2} \mathrm{Cr}_{2} \mathrm{X}_{4}\left(\mathrm{M}=\mathrm{Al}^{3+}, \mathrm{Ga}^{3+}\right.$, $\mathrm{In}^{3+}$ and $\left.X=S, \mathrm{Se}\right), \mathrm{GaAs}_{1-x} \mathrm{P}_{x_{0}}$ $\operatorname{In}_{x} \mathrm{Ga}_{1-x} \mathrm{P}, n$-GaAs, graphite, $\mathrm{Si}$ and $\mathrm{Ge}$ crystals prepared by the sublimation, chemical transport and chemical reaction techniques.

Session II. Initial stage and nucleation of evaporated thin films (7 papers)

Growth of $\mathrm{Au}$ films on cleaved $\mathrm{NaCl}$ surfaces and on low-temperature substrates. Structures of CdS, CdSe, $\mathrm{As}_{2} \mathrm{~S}_{2}$ and silver films.

Session III. Growth from fluxes and solutions ( 6 papers)

Growth of $\mathrm{NaCl}$ whiskers and $\mathrm{LilO}_{3}$ crystals from their aqueous solutions, $\mathrm{TiB}_{2}, \mathrm{ZrB}_{2}, \mathrm{MnB}$ crystals from Al fluxes, $\mathrm{Cr}_{2} \mathrm{O}_{3}$ crystals from $\mathrm{K}_{2} \mathrm{~B}_{4} \mathrm{O}_{7}-\mathrm{PbO}$ flux, $\mathrm{ZnS}, \mathrm{CdS}$ and $\mathrm{ZnSe}$ crystals from $\mathrm{Bi}$ fluxes.

Session IV. Melt growth (6 papers)

$\mathrm{BaTiO}_{3}, \mathrm{TeO}_{2}, \mathrm{Ca}_{12} \mathrm{Al}_{14} \mathrm{O}_{33}$, orthoferrite and $\mathrm{Hg}_{1-x} \mathrm{Cd}_{x} \mathrm{Te}$ crystals grown by Czochralski, floating zone and Bridgman methods.

Session V. Whisker (7 papers)

Growth and growth mechanisms of $\mathrm{Te}$, Se, ionic crystals and metals.

Session VI. Texture (7 papers)

The $X$-ray topographic studies of $\mathrm{KDP}, \mathrm{NaClO}_{3}$, quartz, ice, GaAs, Si and Al single crystals.

Sessions VII and VIII. Miscellany (12 papers)

Theories of crystal growth, solid phase reactions, high-pressure and hightemperature techniques, polymer and organic crystals.
Abstract booklet of the conference (110 pages with Figures, diagrams and photographs, written in Japanese) are available from the Japan Society of Applied Physics, Kikai-Shinko Bldg., 21, Shiba-Koen, Minato-ku, Tokyo at 600 yen plus postage.

Department of Physics
Gakushuin University
Mejiro
Tokyo
Japan

T. OgaWA

\section{Conference on Safety in X-ray Laboratories. 13 November 1970.}

The Crystallography Group of The Institute of Physics and The Physical Society (London) devoted its $1970 \mathrm{Au}$ tumn Conference to the topic of Safety in X-ray Laboratories. The meeting was held at the Institution of Electrical Engineers, London, not inappropriately, perhaps, on Friday 13 November. It covered in four sessions all aspects of radiation safety from reiteration of currant thinking on the nature of the dangers presented by crystallographic equipment to the problem of their elimination. There were 207 participants, drawn from practising $X$-ray crystallographers, radiation protection personnel and manufacturers of commercial X-ray equipment. The resulting discussion and comment was wide-ranging and informative.

The first session of the conference consisted of three invited papers on the nature and magnitude of the dangers of currently used crystallographic apparatus, and on the problems of monitoring installations and their users. Much of this material is well known, but it can hardly be over emphasized, especially at a meeting on safety. The opening paper, by Mr M. J. Butler (Radiological Protection Service, Sutton, Surrey), considered the particular dangers of crystallographic as compared with other X-ray equipment: narrow direct beams having dose rates high enough to produce serious tissue damage in a very few seconds; working positions which usually are near to the tube focus; scattered radiation often in the form of narrow beams which may be intense. The organs mainly at risk are hands, face and eyes. Film badges, ring and thermoluminescent dosimeters all too easily escape narrow scattered beams. In these circumstances reliable monitoring of stray radiation and of received doses is not 
easy, and the elimination of stray radiation by the provision of reliable and effective main beam shutters, and by the correct fitting of attachments, becomes vital. Total enclosure, as a means of eliminating routine-operation hazards, becomes tempting, at least to radiation protection officers. In this session two approaches to the problem of monitoring leakage radiation were discussed. One involved an elegant absolute measurement of the dose rate from an $X$-ray beam, described in a paper by $\operatorname{Dr} W$. A. Wooster and Mr G. A. Wooster (Crystal Structures Ltd., Cambridge). This was then applied to the calibration of the optical density of films used to monitor leakage radiation as a function of dose. The other was based on the use of Geiger-Müller $(G-M)$ tube detectors. The speaker, Dr P. W. Roberts (Department of Physics, University College, London W.C. 1), suggested that detection and elimination of a leak is usually required, rather than exact measurement of its dose rate, and showed that a G-M tube, used with a calibration factor derived for $X$-ray energies of about $10 \mathrm{keV}$, gives an adequate measure of dose rate.

The second session consisted of papers by Mr J. M. Rees (Advisory and Information Unit Department of Employment and Productivity, London W.2) and Dr D. Hughes (Radiation Protection Service, University of Leeds) giving some dozen examples of accidents with $\mathrm{X}$-ray crystallographic sets. These accidents varied from alarming but fortunately not serious failure of safety devices to accidents eventually resulting in amputation of finger joints; they involved both old and very new equipment, and operators ranging from research students to experienced service engineers. Perhaps one characteristic of the cases described which should be noted is the large variety of situations which can lead to accidents. Another is the sometimes incredible lack of anticipation by users of the possible consequences of their actions both for themselves and for others - actions such as the modification or over-riding of safety devices so as to enable the attachment of apparatus which would otherwise not fit 'safely'. Possibly this lack of anticipation occurs because familiarity arising from the frequent use of any equipment, including $X$-ray equipment, soon allows its dangers to recede from the forefront of the mind. If this is the case, safety will be achieved only when equipment is made entirely fool-proof or when users are frequently and forcefully reminded of dangers, or both. It seems unlikely that completely fool-proof equipment will ever be available.

The third session was devoted to a large number of short contributions by practising $X$-ray crystallographers who described safety devices which they had themselves made. Lack of space here unfortunately prevents detailed descriptions of them. Many were concerned with the safe attachment of auxiliary equipment to the $X$-ray generator. Two groups may be distinguished: (a) systems in which removal of the attachment causes immediate closure of a primary beam shutter, $(b)$ systems in which a primary beam shutter must be closed before the attachment can be removed. Class (a) includes electrically and mechanically actuated systems, whereas class $(b)$ consists of mechanically actuated systems only. The systems in both classes prevent the primarybeam shutter being opened in the absence of the attachment and all usually have very low-leakage labyrinth connexion of collimator to tube housing. When properly designed and fully implemented they are very safe - though in the previous session an accident arising from a worn mechanical interlock was described - and cut to negligible proportions leakage radiation arising before the collimator exit aperture is reached. They are difficult to design and implement fully in most $\mathrm{X}$-ray laboratories because of the presence of generators and attachments made by a range of manufacturers, but a notable example of a fully implemented system is that in the Crystallography Department, Birkbeck College, London, described by Professor J. W. Jeffrey. This incorporaltes a mechanical system in class $(b)$ above, and is used with the Manchester system of remote, optical camera alignment which was described by Mr W. Hughes (Physics Department, University of Manchester Institute of Science and Technology). This problem of multiplicity of equipment was discussed in the following session as well. All the above systems, however, are ineffective beyond the end of the collimator. Many attachments in current use are open and may emit significant amounts of radiation, e.g. from the specimen but not intercepted by the detector, or by air and back-stop scattering. The elimination of this by good design of the attachments is surely a matter for the manufacturer, but the problem is sufficiently serious with many currently used attachments for users to have devoted much effort to its solution. Several have introduced total enclosure systems in which the tube housing and attachments are entirely enclosed in a box opaque to $X$-rays. This, of course, eliminates leakage radiation from all sources. There was lengthy discussion of the advantages and disadvantages of this arrangement and it is clear that, safe though it might be and attractive to radiation protection officers, many crystallographers are unenthusiastic because it makes setting up and adjustment of attachments difficult and does not prevent accidents during that time.

The final session contained two papers only. The first, given by Miss B. Stern (Radiation Unit, University of Surrey, Guildford, Surrey), reported the findings of a survey recently conducted by the Association of University Radiation Protection Officers into safety of $X$-ray sets in British universities. Approximately 200 sets were covered and the survey examined the existence and nature of warning signals, shutter mechanisms, accident-history and modifications made by users to commercial equipment. The speaker concluded that the time and money spent by users on safety devices is considerable, even, though less, with modern sets. She stated that she was strongly of the opinion that there is great need for attachments and tube housings to have standard connexions, i.e. for the design of an internationally agreed standard interface. It is clear that it would be difficult to arrange and would still not solve all problems, but the gains would be so great as to justify a large effort to this end. The final paper in the conference was given by Drs V. L. de Vries (application Laboratory, P. I. T. Products Division, N. V. Philips Gloeilampenfabrieken, Eindhoven, The Netherlands) who presented the manufacturers' viewpoint. Drs de Vries discussed the difficulties which manufacturers have experienced from changes in protection standards over the past few years and remarked that many users are reluctant to pay the monetary and restricted-operation cost of increased safety so that each manufacturer makes his own compromise. $\mathrm{He}$ welcomed the idea of a standard interface, provided it were carefully designed and international, but insisted that its introduction would present great difficulty. In any case development times of $X$-ray equipment are of the order of five years and standardization would inevitably be a long-term solution.

This meeting showed very clearly that most crystallographers are sensible and responsible people, aware of the dangers of their equipment and active in 
taking steps to prevent accidents. Much effort has been made to reduce the dangers, and most of this has been made by crystallographers themselves. These efforts have resulted in a low accident rate, but that is little consolation for the recipient, for whatever reason, of an avoidable dose of radiation. Serious accidents still occur. Other groups than crystallographers are concerned about the safety of X-ray crystallographic equipment and it is clear that crystallographers will not be allowed to go to the devil in their own way. Differences of approach to safety probably exist between the various parties involved and if crystallographers are to avoid having unacceptable safety controls imposed upon them they must ensure that they take the lead in any discussions of the safety of their equipment and proce. dures.

$$
\begin{aligned}
& \text { R. HINE } \\
& \text { (Conference Secretary) }
\end{aligned}
$$

Physics Department

University College

Cardiff

Wales

\section{International Conference on Current Research in X-ray Crystallography. Manches- ter, 14-16 April 1971.}

The Spring and Autumn conferences of the Crystallography Group (formerly XRAG) of the Institute of Physics and the Physical Society are well known to many continental crystallographers in Europe. This year's Group Spring conference had been organized jointly with the Chemical Crystallography Group of the Chemical Society, on behalf of the U.K. Crystallographic Council. Moreover it was an international meeting: most of the 10 invited speakers were non-U.K., and there was a discussion, on the first night, about the possible formation of a European Crystallographic Association. This well-attended discussion was useful in that the attitude of representatives, both of various European countries and of the International Union, was clearly stated. No further steps are at present envisaged, but a very loose form of contact has been established between representatives of the national unions in Europe.

It is impossible even to enumerate here the 45 or so papers, ranging from crystal structures and methods of anal$y$ sis to a great variety of miscellaneous subjects; so we can only give a few impressions. From the papers concerned with structure analysis, two remarks are worth mentioning here because they drove home very effectively the awareness that all is not pour le mieux dans le meilleur des mondes' regarding the analysis of structures even of moderate complexity, which nowadays is often presented as a next-to-routine business.

First: In her invited paper Accurate Structure Determinations, Professor A. Vos (Groningen) pointed out that an important category of analyses of organic compounds aims at results interpretable in terms of existing valence theories, but very few of these investigations are carried out at the low temperature required to reduce sufficiently the uncertainties due to thermal diffuse scattering.

Second: Mr Gilmore and Dr Woodward (Bristol) illustrated Problems of pseudo-symmetry by a 'simple' structure which nevertheless could be solved only by - be it sophisticated - trial and error procedures. It contained light atoms, plus gold in a special position. Most crystallographers who have encountered similar situations will prefer to forget the frustrating efforts they spent on them, either with or without success. So it is a good thing to be reminded that there are still problems asking for a systematic solution.

Some papers merely presenting and discussing results of structure analysis were not read, but were presented in a 'poster session'. Each author had a stand on which drawings, tables, models etc. were exhibited. Interested participants were invited to discuss these results privately with the authors. Judging both by the lively discussion groups formed and by the favourable opinions heard from some of the authors, this way of dealing with subjects of interest to only a limited number of participants was very successful.

As usual in this type of conference, the time available for discussion of the papers was very short. An exception had been made for a paper (by S. H.
Banyard and P. R. Evans, Oxford) on the assessment of a rotating-drum type microdensitometer and on the possible centralized service to be provided by an institute using this instrument with an on-line connected computer. The idea was that people could send in their diffraction films and receive a tape containing the complete list of integrated intensities. The discussion revealed that such a procedure might not be free from communication troubles. Moreover the results will suffer from the accuracy limits imposed by the photographic process, as compared with counter diffractometers. On the other hand, the latter are apparently not considered as an economical solution for at least some types of laboratories, so the microdensitometer may still have a future in crystallography.

One morning was devoted entirely to the teaching of crystallography. In this session a paper on A Programmed Course in Crystallography by $\mathrm{Dr} \mathrm{H}$. Schenk c.s. (Amsterdam), drew much attention. The 3 week course in question has been given for a year now It deals with a considerable number of subjects in crystallography and structure analysis. The authors are enthusiastic about the speed at which students learn in this way, as well as their eagerness in doing so, especially when compared with courses of the conventional type.

The conference was very well organized. One feature of many former Group Meetings was retained: the evening leciure. This one was on Reminiscences of a crystallographer at Manchester and Cambridge, by Professor H. Lipson. 'People had more fun doing research and cared less about motives in pre-war days', he told us. The first part of this statement was amply and most convincingly proven. Also it became quite clear that pre-war workers were neither unmotivated nor carefree. Accordingly, Professor Lipson's lecture was appreciated by the audience as a major contribution to a very stimulating conference.

P. M. DE WOLFF

\author{
Laboratorium voor Technische \\ Natuurkunde \\ Technische Hogeschool Delft \\ Delft \\ The Netherlands
}

